\title{
UN LENGUAJE COMÚN PARA ENFERMERAS: DILEMA PERSISTENTE
}

María da Gloria Miotto Wright *

La autora busca mostrar en el transcurrir de la exposición que el tema como fue solicitado para ser desarrollado, presenta una perspectiva que no es la más apropiada. Para justificar esa afirmación la autora utiliza el modelo operacional de la teoría crítica aplicado a la salud. El modelo analiza aspectos de la ideología del tema; los factores que han contribuido para que el dilema persista dentro y fuera de la profesión; la visión general de la realidad de América Latina y indica las acciones transformadoras necesarias para discutir y trabajar el tema.

Unitermos: Lenguaje común, dilema persistente, modelo operacional, teoría crítica, ideología, acción transformadora

Quisiera agradecerles la invitación para participar de este I Foro Científico Nacional de Enfermería Materno Infantil. El tema que solicitaron que yo desarrollara, "Un Lenguaje Común para Enfermeras: Dilema Persistente", es un desafío.

Quisiera aprovechar la ocasión para presentarles algunos de mis indagaciones y describirles los problemas que llevan al dilema. Iniciaré por el significado del tema y su ideología, en el contexto de la política social y de salud. Aplicaré la teoría crítica y el modelo operacional que desarrollé para la salud (WRIGHT, 1990) al análisis del pensamiento crítico y de la construcción de una acción.

El proceso de escribir sobre un tema no es solamente un acto narrativo. Al percibir el tema como un fenómeno situado en una realidad concreta mediada por, personas, yo debo escribirlo teniendo una actitud crítica y transformadora.

*Profesora Adjunto del Departamento de Enfermería/Faculdade de Ciencias de la Salud Universidad de Brasilia/Brazil; Consultora Internacional/PAHO/WHO/WASHINGTON 
Los participantes de ese Foro también deben tener una actitud semejante para lograr los objetivos de esta reunión. En tratar de conocer el tema, vamos evitar ideas preconcebidas. Yo no vengo a traerles "la verdad", pero si a buscaría basado me en los hechos y plantear muchas preguntas que tendremos que responder.

Revisé la literatura de enfermería y los diccionarios Webster's y Pequeño Larouse para las conceptualizaciones que estaré utilizando.

Los pasos están ilustrados en la Figura 1.

\section{I - EL SIGNIFICADO DEL TEMA}

El avance de la ciencia y de la tecnología hace necesario especificar los conocimientos, normas, patrones de una determinada área científica.

Para entender la esencia del tema Un Lenguaje Común para Enfermeras: Dilema Persistente, es necesario analizar sus componentes principales. El término Lenguaje Común puede ser conceptualizado como "El medio sistemático de comunicación de 10 que pertenece, es conocido y compartido por los miembros de una clase o profesión".

Si ya tenemos un lenguaje común, la enfermería divide con las otras áreas científicas una comunicación sistematizada, clara y precisa refleja los valores, cultura y avance de la profesión. ¿Esto significa que la profesión ya contestó las preguntas básicas:? "Quién Somos", "Qué Queremos", "Como Queremos", "Para Quién Queremos" y "Cuándo Queremos?"

Si la Enfermería no tiene un lenguaje común, es necesario preguntar:

- “¿Porqué estamos aquí discutiendo ese tema?"

- “¿Cuáles las inquietudes de la Enfermeras en relación al tema?"

Al analizar un tema y proponer una acción, reconocemos la esencia del tema y somos obligados a aprenderlo como un desafío histórico para la Enfermería. Resulta difícil permanecer neutros. Tenemos que analizar la ideología del tema y buscar el conjunto de ideas, principios y estrategias que se relacionan con el tema Lenguaje Común para Enfermeras.

\section{II - LA IDEOLOGÍA DEL TEMA}

Ideología puede ser concebido como Los principios integrados, las teorías y las estrategias utilizadas para ofrecer cuidados a la población, constituyendo el programa de acción de la profesión de Enfermería.

De esta forma, Lenguaje Común pasa a ser una de las estrategias utilizadas 
para ofrecer cuidados a la población, llevándose en consideración su grado de excelencia. Es necesario tener un patrón que regule la calidad de excelencia, lo que, $t$, en el mercado de trabajo se llama Control de Calidad.

La ideología de la estrategia de Lenguaje Común incluye los principios del Control de Calidad, se pretende asegurar adecuada especificación del tratamiento o cuidado ofrecido a la población por una determinada profesión e instituto de salud.

Asegurar la calidad en Enfermería implica que el nivel de calidad puede ser definido y asegurado al público (HODGES \& ICENHOUR, 1990, p. 242). Es fundamental reconocer que la calidad de la práctica de Enfermería está relacionada con cantidad y costos.

Según ANA (1980, p. 2) "el control de calidad incluye atención a la cantidad del cuidado (utilización) y el control del costos para que el paciente reciba solamente los cuidados necesarios, ofrecidos a bajo costo y compatibles con la calidad".

EL CONSEJO INTERNACIONAL DE ENFERMERAS (1991) afirma que La Garantía de Calidad está basada en las normas y métodos de evaluación del ejercicio, servicio y enseñanza de la enfermería.

Para tener la ideología de control de calidad se exigen los siguientes elementos:

- Liderazgo

- Conocimiento Científico

- Reglamentación de la profesión

- Desarrollo del diagnóstico de Enfermería

\section{LOS ELEMENTOS DE LA IDEOLOGÍA DEL CONTROL DE CALIDAD}

Necesitamos, entonces, averiguar cómo está nuestra profesión en relación a estos cuatro elementos.

\subsection{Liderazgo}

Enfrentamos una situación de liderazgo débil en Enfermería por motivos relacionados al valor de la mujer en la sociedad y a la educación de la enfermera, que se desarrolla como elemento no facilitador de la formación del líder y de responsabilidad.

\subsubsection{La mujer en la sociedad}

La falta de reconocimiento del valor de la mujer en la sociedad se refleja en 
la Enfermería, profesión cuyos miembros son predominantemente del sexo femenino. A pesar del espacios que la mujer ha conquistado en la sociedad, todavía hay dificultad en conciliar los papeles domésticos tradicionales (ama de casa y socializadora de los hijos) y sus papeles profesionales. La mujer se mantiene alejada de las decisiones y enfrenta discriminación en el trabajo, en la educación y en la política (WRIGHT, 1984, p. 341).

Los espacios que la mujer ha ocupado en la sociedad no fueron dictados por ella. Aún hoy día, las mujeres no tienen una solidariedad orgánica en que se basa toda una comunidad unificada (BOUVOIR, 1980, p. 363).

Para haber transformación en la profesión de enfermería, la mujer tiene que transformarse, tiene que ser recreada por si misma. No basta modificar las situaciones económicas para que la mujer se transforme. Libertar la mujer tradicional y profesional es concientizarla de su naturaleza, conocer sus potencialidades y saber usar los recursos que la sociedad y el medio ambiente ofrece.

\subsubsection{La educación de enfermería}

Tanto en los países desarrollados como en desarrollo la educación profesional es influenciada por los acontecimientos y crisis en las esferas política, socioeconómica, y salud. Todavía está bajo el dominio clínico/hospitalario y de los modelos médicos. Esta situación contribuye para la división entre la enfermería académica y la enfermería clínica, perdiendo la visión de conjunto.

La enfermería académica procura proveer una respetable y efectiva educación para sus estudiantes y también desarrollar bases teóricas y científicas para la enfermería práctica. La enfermería clínica, por su parte, quiere ofrecer un servicio de enfermería efectivo y económico para sus pacientes. Las estudiantes reciben un curso teórico sin mucha aplicación práctica y entrenamiento práctico sin mucha base científica. En dicha situación se pierde la perspectiva de la formación del liderazgo de la profesión.

Varios modelos ya fueron diseñados para quebrar esa dicotomía, incluyendo: estructura organizacional, financiamiento conjunto, docente en la práctica, experiencias del campo clínico para el estudiante, transición del papel de estudiante recién-graduado para enfermera clínica. Pero el problema todavía no es resuelto.

El ingreso de las enfermeras en la carrera universitaria pasa exigir una formación científica a nivel de especialización, maestría y doctorado. Es necesario superar el modelo curricular del enfoque enfermedad bajo el modelo de la enseñanza médica.

A pesar de haber experiencias aisladas con modificaciones en el currículo de enfermería, su impacto en la transformación de la profesión como un todo ha sido muy limitado.

Los modelos norteamericanos, canadiense y europeo de la educación y práctica de enfermería han sido importados por los países en desarrollo, sin hacer 
antes un estudio de factibilidad de su aplicación y los beneficios que proporcionarían. Ejemplos incluyen el uso de libros textos extranjeros, principalmente los del área básica, como única fuente de aprendizaje de la enfermería, y la incorporación de las teorías de enfermería en la enseñanza sin hacer un análisis crítico de su viabilidad en el país.

Necesitamos desarrollar modelos propios y modificaciones en la enseñanza que promuevan liderazgo.

\subsection{Conocimiento científico}

Según enfermeras, el término "Conocimiento Científico" no ha sido conceptualizado claramente. La mayoría de los libros definen el término "Conocimiento" separadamente del enfoque científico. Las discusiones sobre este as unto son sobre el conocimiento específico de la Enfermería.

En Enfermería, entiendo que el Conocimiento Científico es la Condición de conocer alguna cosa con familiaridad adquirida a través de experiencia y asociación, generando un conjunto de conocimientos que exhibe los métodos o principios de la ciencia.

La Enfermería ha sido criticada por no tener un cuerpo único, definido, de: conocimiento. En décadas recientes, la Enfermería ha hecho algún progreso en esa dirección. Lo que se ha producido como Conocimiento Científico en los países: desarrollados es derivado de las investigaciones, de las teorías y del uso de esa producción en la práctica.

En contraste, en los países latinoamericanos, la producción del Conocimiento Científico proviene de tesis de postgrado o del concurso a la carrera de magisterio, de investigaciones de docentes y de proyectos encomendados a las asociaciones de clase. Hay poca participación de las enfermeras de la práctica. Tampoco hay desarrollo de teorías propias.

Al revisar la definición y redefinición del conocimiento de Enfermería en los Estados Unidos y Canadá, encontramos algunas estrategias para desarrollar el conocimiento científico en Enfermería, tales como:

a) El uso de pensadores apoya, desafía y ofrece nuevos horizontes en la producción del conocimiento científico;

b) Desarrollar un conjunto mínimo de datos para establecer padrones uniformes para la colecta de datos comparables, mínimos y esenciales en Enfermería;

c) Desarrollo de programas de computadores para analizar datos complejos, problemas de diagnóstico o sugerir el mejor camino. Ellos ofrecen conocimientos para toma de decisiones;

d) Organización del conocimiento científico de Enfermería en términos de sus áreas de concentración o prioridades de investigación. (McCLOSKEY \& GRACE, 1990, p. 42). 
Se requieren el desarrollo, la diseminación y la implementación del conocimiento científico de Enfermería en la práctica profesional para obtener su reconocimiento por otras áreas del conocimiento científico, dentro y fuera de la salud.

\section{3 - La reglamentación de la profesión}

De forma general, las profesiones funcionan por intermedio de una especie de "contrato social" entre ellas y la sociedad. La Enfermería tiene la obligación de autor reglamentarse para asegurar la calidad de servicios vitales a la sociedad.

La Reglamentación profesional consiste en las modalidades mediante las cuales se ordena, se da coherencia y se controla una ocupación y su ejercicio (CIE, 1991, p.5). La reglamentación puede presentarse a veces sutil y otras veces audaz; directa o indirecta; ocurre en muchos ámbitos y en niveles múltiples. Las dimensiones de los sistemas de reglamentación son los propósitos, objetivos, mecanismos, autoridades, agentes, normas, métodos e instrumentos.

Un sistema de reglamentación de la profesión debe comprender:

- "Normas elevadas para el desarrollo personal y profesional y para el desempeño de las enfermeras;

- Aprobación pública para que las enfermeras se desempeñen conforme a sus capacidades;

- Participación de la profesión en la formulación de políticas públicas;

- Responsabilidad de la profesión ante el publico por las funciones que desempeñe en su beneficio; $y$

- Reconocimiento y remuneración adecuados por las contribuciones de la profesión, así como oportunidades para que sus miembros puedan mantenerse siempre al día", (CIE, 1991, p. 26)

EI CIE también considera que pueden afectar el proceso de reglamentación los factores que se mencionan a continuación:

- "La calidad y los objetivos de los servicios;

- La accesibilidad y el costo de los servicios;

- Las políticas públicas (salud, educación, trabajo, bienestar social, economía);

- Otros trabajadores de la salud;

- Los objetivos de la profesión; y

- La posición y el bienestar social de los profesionales." (CIE, 1991, p. 83)

Estos factores tienen efectos directos sobre:

- La estructura de la profesión, es decir, las categorías del personal de enfermería;

- El ámbito y ejercicio de la profesión;

- Normas de enseñanza y de práctica;

- Estructura de responsabilidades; y

- Formas y control de la reglamentación. 
El Consejo Internacional de Enfermeras (CIE) ha sido el portavoz de la Enfermería en el ámbito internacional sobre la reglamentación y viene asesorando a las Asociaciones Nacionales de Enfermeras (ANEs) en el camino para lograr sistemas óptimos de gestión profesional en sus respectivos países.

El empeño del CIE en liderar el movimiento de reglamentación de la .profesión se da principalmente en virtud de la presión de los siguientes factores sociopolíticos:

- "Ampliación del papel de Enfermería en conformidad con el mandato mundial de atención primaria de salud;

- Respuestas a las complejidades de los cuidados médico-asistenciales ocasionados por las nuevas tecnologías y por los sistemas y problemas cambiantes;

- Emergencia de nuevas categorías de trabajadores de salud;

- El movimiento de especialización estaba cobrando ímpetu;

- Una demanda grande de enfermeras y la escasez de personal calificado en el mercado;

- Redefinición de las fronteras de la Enfermería en relación con otras profesiones de la salud; $y$

- Crear nuevas oportunidades y lograr mayores incentivos y más prestigio para las: enfermeras." (CIE, 1991, p. 2).

La reglamentación de una profesión no es responsabilidad solamente del CIE. Es una responsabilidad mucho mayor de sus miembros y de las Asociaciones Nacionales de cada país. Es un compromiso con la profesión que eligieron seguir.

\section{4 - Desarrollo del diagnóstico de enfermería}

La práctica de Enfermería involucra la interacción con las otras profesiones de la salud que prestan asistencia a los pacientes. A veces la enfermera advierte sobre problemas del paciente que deben ser tratados por otros, y ignora o deja de detectar problemas que ella propia puede tratar.

La Enfermería necesita un sistema de clasificación para organizar sus funciones y definir su esfera de acción. EL DESARROLLO DEL DIAGNOSTICO DE ENFERMERÍA es una parte integral del desarrollo de Enfermería como profesión. Es importante usar el DIAGNOSTICO en la práctica de enfermería para ofrecer y mantener la calidad de los servicios (CARPENITO, 1987, p. 3).

Yo considero que el DIAGNOSTICO DE ENFERMERÍA es "la decisión o opinión resultante de un examen o investigación sobre el estado de salud de una persona. EI Diagnóstico es parte integrante del PROCESO DE ENFERMERÍA."

Como no hay un lenguaje para diagnóstico de enfermería, sus cuidados son descritos en términos de la taxonomía médica de las enfermedades, especialmente en relación a los cuidados intensivos.

Las ventajas del desarrollo de un sistema de clasificación o taxonomía. Para enfermería son las que siguen: 
- Un sistema de clasificación permitiría desarrollar los fundamentos científicos sólidos que enmarcan una profesión;

- Una única taxonomía daría un marco común de referencia para los cuidados, la educación y la investigación en enfermería;

- Una terminología sólida facilitaría la comunicación oral y escrita, haciéndola más eficaz;

- Establecería un sistema adecuado para el empleo del tratamiento informático; y

- Integraría las actividades de enfermería relacionadas con el proceso y diagnóstico en el sistema de información de la asistencia a la salud.

El desarrollo de tal taxonomía en enfermería está apenas empezando. Las enfermeras norteamericanas han avanzado más en este sentido. Ellas han utilizado en método inductivo, saliendo de observaciones específicas hacia generalizaciones. Enfermeras con diferentes experiencias clínicas y educativas colaboran para identificar y describir problemas de salud que las enfermeras diagnostican y tratan. Estas enfermeras recogen de sus experiencias y de la literatura pertinente fenómenos clínicos que describen diversos estados de salud. Este método produce categorías generales de diagnósticos que pueden ser utilizadas por la mayoría de las enfermeras.

Dicho sistema de clasificación tiene que ser relativo a las funciones y responsabilidades exclusivas de la enfermera. El desarrollo de categorías específicas del diagnóstico constituye elementos del sistema de clasificación. Ellos representan una clase de problemas de salud que tienen el mismo nombre y la misma definición de características (signos y síntomas), y son consideradas equivalentes.

Actualmente las categorías diagnósticas de enfermería son clasificadas en orden alfabético. Una taxonomía es construido por las dimensiones horizontales y verticales de un sistema de clasificación. Cuando una taxonomía se desarrolla, las categorías se relacionan entre si por el hecho de estar en el mismo nivel de una clase abstracta. Las categorías deben ser las más genéricas posibles para representar el mundo real de la práctica de enfermería. Cuánto más abstractas las categorías, más distantes estarán de la práctica de enfermería.

Para que un sistema de clasificación sea aceptado y usado, tiene que haber un programa de investigación para identificar los siguientes ítems para cada categoría del diagnóstico:

1) el prototipo de la categoría a un nivel de generalización útil;

2) los criterios de diagnóstico que tiene los niveles más altos de validad; y

3) las diferencias de los criterios de diagnóstico que permiten discriminación entre categorías. (GORDON, 1985, p. 47)

Actualmente hay críticas fuertes en relación al sistema de clasificación por el Grupo Nacional para la Clasificación de Diagnósticos de Enfermería de los Estados Unidos. Se dice que el sistema es muy complejo y tiene un "lenguaje esotérico", y que ciertos conceptos de diagnóstico no son aceptables filosóficamente. Algunas enfermeras dijeron que el sistema de clasificación es demasiado extenso o 
demasiado restrictivo y que ciertos diagnósticos son demasiadamente médicos.

La decisión de desarrollar un sistema de clasificación para la práctica de enfermería está relacionada con el contexto socioeconómico y político del país, su nivel de desarrollo allí, el uso del proceso de enfermería en la práctica profesional y como el diagnóstico será utilizado en el modelo de referencia de la prestación de cuidados de enfermería a la población.

\section{IV - CONTINUIDAD DEL DILEMA}

El "Dilema" existe porque las enfermeras todavía no están completamente conscientes de su relación con la sociedad. No consiguen percibir claramente los cambios reales o los perciben de una forma errada. Los datos que dominan su conciencia son solamente los de la experiencia vivida. Este tipo de conciencia no consigue objetivar los hechos y situaciones problemáticas de la vida (FREIRE, 1985, p. 75-77).

El dilema dejará de existir cuando las enfermeras sean comprometidas con la profesión y del mundo actual. Esto implica tener una conciencia crítica de la realidad para saber proponer acciones que cambien las estructuras cerradas de la profesión y del sistema de salud del país. Estas acciones precisan ser transformadoras en la forma de participación, toma de decisiones, y de liderazgo, de ofrecer una educación libertadora en la formación de las enfermeras y en el desarrollo del conocimiento científico.

\subsection{Factores que dificultan un lenguaje común}

\subsection{1 - Factores fuera de la profesión de enfermería}

- Ambigüedades y Crisis de las Políticas Sociales y de Salud

En América Latina, el sector público-y más específicamente en el sector social-existe una falta de racionalización en las decisiones para distribuir recursos. El proceso es complejo y poco transparente por la variedad de actores, fuerzas sociales y políticas involucradas y por la falta de experiencia histórica democrática.

Existe una ambigüedad en relación al término "la política", que se entiende de acuerdo con las distintas disciplinas del conocimiento. Para los economistas significa análisis racional, mientras los cientistas sociales lo entienden más asociado y dependiente de los procesos políticos, por lo tanto, con una racionalidad diferente (MAINGÓN, 1992, p. 2). 
Esa ambigüedad se refleja también en el desarrollo de la profesión de medicina y de enfermería. La racionalidad que se aplica en un sector no es necesariamente lo que se usa en otro contexto de referencia. La falta de una instrumental técnica racional nos deja sin capacidad de seleccionar buenos y adecuados medios para resolver los complejos problemas sociales y de salud. Esto genera crisis constantes en estos sectores de la sociedad y en la práctica de enfermería. Un ejemplo de la interferencia de las crisis políticas en enfermería es la elaboración de los programas de atención a grupos específicos, como Materno-Infantil. Frecuentemente los programas propuestos no se implementan o se suspenden debido a falta de recursos.

Todas las políticas tienen contenido ideológico y normativo. Se precisa reconocer dentro de estos elementos la racionalidad comunicativa (HABERMAS, 1974, p. 49-55; 1979, p. 50) para tomar decisiones estructuradas y apoyadas en la interacción cooperativa. De otra forma, la implementación del programa político depende de interés personal.

La ambigüedad y las frecuentes crisis del sector político conlleva a una comunicación deficiente entre los diferentes sectores de la sociedad y de las profesiones. Esto proporciona una práctica de dominio y opresión y impide una práctica colaborativa y participativa.

La política será desvirtuada por intereses personales y dominio dentro de las relaciones profesionales cuándo las políticas sociales y de salud son elaboradas sin tomar en cuenta los valores y creencias que determinan los patrones normativos de comunicación.

- Intereses del Sistema de Salud y el Desarrollo de la Enfermería

Hay conflictos de intereses que se relaciona con dos aspectos que seguidamente se mencionan:

- Las diferencias relativas a los aspectos económicos y los valores profesionales, o sea, la necesidad de economizar versus el compromiso del comportamiento profesional.

Lo que significa buscar nuevas estrategias para asignar los recursos escasos para la salud y la Enfermería en beneficio del paciente;

- La creación de competencia entre y dentro de las profesiones de salud cuando se redefinen los papeles se redistribuye el poder. Este conflicto está relacionado con: la expansión del papel profesional de enfermería; una preparación educacional apropiada para empezar la práctica profesional; y realocación del poder dentro del sistema de asistencia a la salud.

\subsection{2 - Factores dentro de la profesión}

- Consideraré apenas dos factores esenciales y interrelacionados: 
- Ausencia de un Modelo Propio de Enfermería para América Latina

Dentro de las profesiones de la salud, es la enfermería que tiene el mayor número de personal para trabajar en la asistencia y cuidados del paciente. Sin embargo, no ha logrado desarrollar un modelo propio de práctica de enfermería, que resaltara sus características específicas, y produjera un impacto dentro del sistema de salud.

Para crear un modelo propio de enfermería en cada país, las enfermeras tendrán que solucionar conflictos como: diversidad cultural dentro y fuera de la profesión; la discriminación socio-cultural; como seleccionar el enfoque teórico más adecuado para permitir la unificación de la enseñanza, etc.

- Poca investigación en Relación a las Necesidades

Los factores responsables por la poca investigación de enfermería incluyen las que siguen:

- Falta de claridad sobre lo que es una investigación de Enfermería;

- Dudas sobre la naturaleza o ámbito de las investigaciones;

- Falta de valoración de la investigación dentro de la profesión, de los Centros de Investigación y de los órganos de financiamiento.

En años recientes, varias enfermeras han tratado de superar la falta de claridad sobre la esencia de esa investigación, produciendo escritos sobre su definición, ámbito y dominio.

En 1977 la Academia Norteamericana de Ciencias escribió que:

Investigación en Enfermería enfoca le papel de enfermería en la prevención de enfermedad, el cuidado del enfermo y la restauración de la salud. Ella está basada en la utilización de información y metodologías de las ciencias biológicas y de comportamiento. Ella es distinta de las otras áreas científicas en su enfoque en el cuidado de enfermería. (BLOCH, 1985, p.125)

La ASOCIACIÓN DE ENFERMERAS AMERICANAS (1980) ha presentado la siguiente definición:

Las investigaciones de Enfermería desarrollan conocimientos sobre; la salud; la promoción de la salud durante toda la vida; el cuidado de las personas con problemas de salud y incapacidades (particularmente para viejos, enfermos crónicos, y pacientes en fase terminal de enfermedad). Este enfoque complementa la investigación biomédica, la cual estudia las causas y tratamientos de las enfermedades. (BLOCH, 1985, p. 25)

En resumen, investigación es la tentativa de solucionar los problemas de una forma sistemática y controlada para poder predecir y explicar lo que se está estudiando. Su producto es el conocimiento y hay que posibilitar la construcción de la "ciencia de enfermería". Las cuestiones principales se refieren al hecho de ser o no esa ciencia única de enfermería, o si la enfermería irá siempre depende únicamente de conocimientos de las otras ciencias. 
Las dudas sobre la naturaleza de las investigaciones resultan difícil clasificar las investigaciones que puedan direccionar las prioridades de la población y de la profesión. En la literatura norteamericana no he encontrado el término "Línea de Investigación". Según POLIT \& HUNGLER (1983, p. 7-14), las investigaciones americanas se basaron inicialmente en educación de enfermería y administración y solamente en años recientes procuraron bases científicas del conocimiento para la práctica de enfermería.

En América Latina la investigación está en sus pasos iniciales, caracterizándose por trabajos aislados, mayormente sobre el aspecto clínico y en las áreas de educación y administración. Son pocos los proyectos metodológicos para elaborar programas y verificar métodos de trabajos y el cuidado de enfermería. Un grupo de enfermeras brasileñas se reunieron y presentaron el siguiente concepto de línea de investigación (CNPq, 1982, p. 176):

Una propuesta de investigación sobre uno o varios temas correlacionados, continuo a lo largo del tiempo, en cuestiones y problemas relativos a las necesidades de la población, realizada de modo progresivo y permitiéndose la incorporación de investigadores nuevos.

La clasificación propuesta incluyó las siguientes áreas y líneas de investigación:

Área Profesional, con las siguientes líneas:

- La enfermería como práctica profesional; y

- Formación de Recursos Humanos en Enfermería.

Área Asistencial, con las siguientes líneas:

- Fundamentos de la Asistencia, Tecnología y Instrumentación;

- Auto-Cuidado a la Salud;

- Riesgos de la Asistencia de Enfermería; y

- Determinantes del Proceso Salud-Enfermedad.

Área de Estructura, Organización y Funcionamiento de las Instituciones, con las siguientes líneas:

- Modelos de la Asistencia de Enfermería;

- Oferta, Accesibilidad y Utilización de los Servicios de Salud;

- Sistema de Información en Enfermería;

- Incorporación de los Conocimientos a la Práctica Profesional; y

- Dinámica de las Organizaciones.

Sin embargo, otro estudio (ASOCIACIÓN BRASILEÑA DE ENFERMERÍA /ME/CAPES/CNPq, 1989, p. 48) indicó que las líneas en Brasil estaban siguiendo otro proceso de desarrollo:

- Núcleo de aglutinación por objeto de investigación (p. ejemplo, la lactancia materna reúne varios investigadores con diferentes teorías, pero todos enfocan el mismo objeto de estudios;

- Núcleo de aglutinación por fundamento teórico (p. ej., los estudios fenomenológicos, materialismo histórico y estudios críticos reúnen varios investigadores que enfocan 
diferentes aspectos de la asistencia de enfermería);

- Núcleo de aglutinación de instrumentación o tecnología (p. ej., la informática) agrupa a investigadores con diferentes objetos de investigación y diferentes teorías).

En 1991-92 otro grupo de enfermeras latinoamericanas se reunieron bajo la OPS/OMS (1992, p.14) y propusieron la siguiente clasificación para investigar en enfermería con enfoque resolutivo:

. El Proceso de Trabajo de Enfermería;

. Estructura y Características de los Servicios;

- Características del Conocimiento y de la Práctica de Enfermería;

. Políticas de Salud, de Educación y de Su Recuperación en la Enfermería;

. Condiciones y Determinantes de la Salud.

El estudio sobre la investigación en Enfermería, que cubrió siete países latinoamericanos y fue coordinado por la OPS/OMS $(1989,20)$ mostró el siguiente cuadro:

. Investigación limitada, con poca capacidad generalizadora, pobremente apoyada y poco diseminada;

. Énfasis clínico con predominancia del individuo como objeto de estudio, dejando de lado la familia y la comunidad;

. Las investigaciones referentes a materiales y tecnologías son bastante incipientes;

- Las investigaciones son en su mayoría de carácter descriptivo y demuestran la influencia de las corrientes positivistas;

. Falta difusión y implementación de los resultados.

Vemos dos extremos: a veces la investigación de Enfermería es tan general que no se distingue de otras áreas del conocimiento científico; otras veces es tan específica a una peculiaridad de la profesión que pierde el contexto de las necesidades de la población. Precisamos de un equilibro, porque la finalidad de la enfermería es promover el cuidado de la salud de la población durante el ciclo vital.

El tercer problema con la investigación es su poca valorización dentro de la profesión y de los órganos de financiamiento. Esto ocurre porque la investigación todavía no está adecuadamente institucionalizada en las Escuelas de Enfermería y dentro de las Universidades. La mayoría de las escuelas no tienen un tiempo destinado exclusivamente a la investigación. Las enfermeras que investigan lo hacen dentro de su carga normal de actividades didácticas, clínicas y administrativas. Así, muchas pierden el incentivo y no se envuelven en investigación. Otras enfermeras, no están preparadas para el proceso de investigación y los métodos estadísticos. Otros obstáculos incluyen: investigaciones limitadas a las escuelas de enfermería con programas de postgrado; falta de acceso a bibliografía actualizada; y salarios bajos.

La dicotomía entre la enseñanza y la práctica conlleva a la poca utilización de los resultados de las investigaciones en los servicios de enfermería. La Enfermería es considerada por la comunidad científica como una "Área Emergente" porque 
ha producido poco conocimiento y este conocimiento todavía no ha valorizado por la sociedad.

Muchos países no tienen una política de investigación en Enfermería y consecuentemente hay pocas enfermeras investigadoras. Algunas excepciones incluyen el Centro para Investigación en Enfermería en los Institutos Nacionales de Salud de los Estados Unidos (1985) y la presencia de la enfermería en el CONSEJO NACIONAL DE CIENCIA y TECNOLOGÍA/CNPq/ Brasil (1986).

El dominio por los médicos, que existe en la salud de una manera general, persiste en los centros de investigaciones. La enfermera tiene que luchar para conquistar espacios en la comunidad científica.

El financiamiento es repartido por las organizaciones financiadoras de acuerdo con los siguientes criterios:

- prioridad del tema dentro de la política de desarrollo de la ciencia y tecnología del país;

- claridad de la presentación del proyecto prepuesto dentro de los pasos científicos de investigación;

- calificaciones del investigador;

- valorización del área dentro de la organización financiadora;

- recursos financieros solicitados de acuerdo con los recursos disponibles;

- recomendación favorable de los revisores del área científica y del comité general.

La gran mayoría de los proyectos de enfermería no consiguen pasar por todos estos criterios o no tienen acceso a la información sobre como concurrir a ayuda financiera.

\section{V - EL CONTEXTO DE LA REALIDAD SOCIAL, DE SALUD Y DE ENFERMERÍA}

Ahora situamos el tema dentro del contexto de la realidad actual latinoamericana para sugerir acciones para transformar la profesión y aumentar su contribución para la salud para la población hasta el año 2000.

\section{1 - El contexto político, social y de salud de la América Latina}

El refuerzo a los procesos democráticos y el la modernización de las relaciones entre el Estado y la Sociedad no ha sido suficiente para terminar el crisis económico y social de los países de América Latina y el Caribe.

Las desigualdades socioeconómicas son profundas y se reflejan en los niveles de pobreza y informalización de la economía (OPS/OMS, 1992, p. 5). El proceso de ajuste económico ha afectado negativamente la oferta y calidad de los 
servicios públicos, en especial los de salud. Hubo, en consecuencia, deterioro de los salarios y un aumento del desempleo (MAINGÓN, 1992, p. 12). Asimismo, se cree que existe un retroceso en el nivel de salud, juntamente con cambios importantes en los perfiles demográficos y epidemiológicos.

Aunque hayan disminuido en las décadas recientes las tasas de fecundidad, mortalidad infantil y muertes por enfermedades infecciosas, se agravaron los factores físicos y sociales y los estilos de vida. Han aumentado los factores de riesgo, como violencias, accidentes, salud mental deteriorada, hipertensión arterial, obesidad, tabaquismo, y el sedentarismo. Se agravan las enfermedades cardiovasculares, el cáncer, SIDA, y lesiones de causa externa.

Simultáneamente, para las personas de bajo ingreso las principales causas de muerte continúan siendo la diarrea, las infecciones respiratorias agudas, las enfermedades vacuno prevenibles y las infecciones endémicas y parasitarias. La pobreza, el analfabetismo y las malas condiciones de vida son el resultado del estancamiento económico del último deceno y originan muchos problemas de salud en la Región.

Los servicios de salud han demostrado insuficiente capacidad institucional para responder a las necesidades actuales y retraso en promoción y prevención. Se privilegia el modelo curativo, con tecnología cara y de uso limitado a pocos pacientes, lo que aumenta los costos sin resolver los problemas prioritarios.

La estrategia y filosofía de "Atención Primaria de Salud" fue implantada en la década de 1980, pero no fue suficientemente fuerte para lograr mejoras en las condiciones de salud de población.

Los órganos internacionales y los Ministros de Salud ya asumieron el compromiso de implementar la nueva propuesta política para de la década del 90 - "La Estrategia de Promoción de Salud" - como una respuesta válida a la situación actual. Esa estrategia apoya los aspectos sociales y ecológicos, como elementos indispensables para lograr la verdadera equidad en salud, incorporando los valores de la medicina social y complementando las estrategias curativa y preventiva.

Esta visión trabaja con el concepto del ser humano como Sujeto Activo, dejando de lado las ideas de Individuo y objeto como receptor pasivo (GOMEZ, 1992, p. 5). Mientras la estrategia de Atención Primaria de Salud estaba basada en el concepto Salud-Enfermedad, la estrategia de Promoción de Salud está basada en el concepto Socio-ecológico de la Salud (OPS/OMS, 1992, p.35).

Según la Carta de Ottawa (OMS/SPBSC/ACSP, 1986, p. 1), Promoción de la Salud es proporcionar a los pueblos los medios necesarios para mejorar su salud y ejercer mayor control sobre si mismo; se complementa esa definición estableciendo que para alcanzar un estado adecuado de bienestar físico, mental y social un individuo o un grupo debe ser capaz de identificar y realizar sus aspiraciones, de satisfacer sus necesidades y de cambiar o adaptarse al medio ambiente. Los prerrequisitos para alcanzar la salud son: la paz, la educación, la vivienda, la alimentación, la renta, un ecosistema estable, la justicia social y la 
equidad. Las líneas de acción para llegar a implementar la Promoción de Salud son: Elaboración de Políticas Públicas Saludables; Creación de Ambientes Favorables; Refuerzo de la Acción Comunitaria; Desarrollo de las Aptitudes personales y Cambios en los Estilos de Vida; Reorientación de los Servicios Sanitarios.

Los indicadores clásicos de salud (que en realidad son indicadores de enfermedades) pasan a ser insuficientes para los actuales desafíos de la promoción de la salud. El nuevo concepto de salud tiene una perspectiva positiva y exige nuevas formas de intervención y modelos e indicadores adecuados.

\section{2 - El contexto de la enfermería en América Latina}

\subsection{1 - Visión general}

La evolución histórica de la Educación de Enfermería en América Latina muestra la influencia del modelo Nightingale, del modelo secular y moderno de la asistencia médica, y de los ajustes correspondientes de las tradiciones de la cultura ibérica.

Los avances incluyen mejoras en la formación científica, con cursos de pregrado y postgrado, y enfermeras en algunas posiciones importantes en las universidades y órganos de educación. Sin embargo, las enfermeras enfrentan los siguientes obstáculos:

- Disminución de la demanda de alumnos para los cursos de pregrado en enfermería;

- Inexistencia de criterios y patrones similares en los distintos países de la Región para implantación, evaluación y cierre de cursos de enfermería;

- Inexistencia de una política institucionalizada de capacitación de los docentes de los cursos de enfermería;

- En algunos países, hay una proliferación desordenada de escuelas privadas de pregrado de enfermería;

- Dicotomía de la enseñanza de pregrado con la de postgrado y de ambos con la práctica profesional;

- Poca preparación de la mayoría de los docentes de los cursos de enfermería con respecto a metodología científica y estadística;

- Poca producción del conocimiento científico, y esta concentrada en los docentes;

- Recursos bibliográficos pobres y desactualizados, y los que hay de origen extranjero;

- Apoyo financiero limitado para las actividades académicas y de investigación;

- Falta de un modelo curricular propio de la enfermería, con dependencia del modelo médico, énfasis en los aspectos clínicos en detrimento de los trabajos en la comunidad, y con referencial teórico en la línea comportamental;

- El paradigma que orienta las actividades académicas y prácticas es "Salud Enfermedad"; 
- Diferencias entre las exigencias del mercado de trabajo y la formación de las enfermeras;

- Estudiantes de nivel socio-económico más bajo que sus colegas de otros cursos;

- Enseñanza poco creativa, creando una baja auto-estima profesional y produciendo una actitud de dependencia y subordinación en el trabajo interdisciplinario;

- Falta de modelos de docentes y líderes profesionales para servir de ejemplos para las estudiantes;

- Falta de conocimiento científico específico de enfermería, obligando la utilización de teorías, conceptos, principios y modelos importados de los Estados Unidos o Canadá.

La situación de la práctica de enfermería en la América Latina sigue las mismas crisis y tendencias de los otros servicios de salud. La enfermería es parte de un trabajo colectivo de promover, prevenir y mantener la salud de la población.

Se registran varias conquistas de la profesión, como su legalización en Consejos o Colegiados Nacionales y Estaduales, sindicalización individual y en conjunto con los otros profesionales de salud, ocupación de puestos en Ministerios y Secretarías de la Salud, creación de las Asociaciones Nacionales de enfermería. Sin embargo, la profesión enfrenta todavía obstáculos como:

- precarias condiciones de trabajo en los servicios de salud;

- falta de un papel definido en la toma de decisiones sobre su propio servicio en la actual estructura de los sistemas de salud;

- bajo salario y pérdidas en la calidad de vida en relación a otras profesiones;

- fragmentación de la profesión y del cuidado de enfermería;

- falta de un sistema de patrón de calidad sistematizado y institucionalizado en la profesión;

- posibilidades de desarrollar iatrogenias;

- un porcentaje grande del personal de enfermería todavía no tiene entrenamiento formal;

- baja satisfacción personal y profesional;

- falta de una carrera con promociones para el equipo de enfermería en los servicios de salud;

- pocas enfermeras son entrenadas en liderazgo y políticas del sector salud;

- falta de un modelo específico de la práctica de enfermería;

- profesión sexo femenino;

- falta de institucionalización del proceso de enfermería en el cuidado del paciente;

- poca aplicabilidad de los resultados de las investigaciones de enfermería en la practica profesional;

- falta de una política de formación y capacitación de los recursos humanos de enfermería en el sistema de salud;

- falta de un trabajo cooperativo entre el médico y la enfermera;

- falta de planeación estratégica en los servicios de enfermería; 
- falta de entrenamiento de las enfermeras de los servicios en investigación y métodos estadísticos;

- falta de financiamiento para investigaciones hechas por las enfermeras de los servicios.

La enfermería latinoamericana necesita participar de un debate continuo, constructivo, transformador para traer nuevas dimensiones a las políticas de salud, asegurando mejor calidad de vida y salud para la población.

\section{VI - LA ACCIÓN TRANSFORMADORA}

Una acción resulta de la toma de una decisión sobre un determinado asunto, programa, política, o cambio de comportamiento. La acción transformadora resulta de la dialéctica entre las condiciones de dominio de la sociedad y la condición o comportamiento de un individuo o grupo dispuesto a conquistar su libertad.

Para llegar a la libertad necesaria para desarrollar un trabajo relacionado con mi tema y el enfoque del Foro, necesitamos de:

1 - Cambiar la perspectiva del tema de Lenguaje Común para Enfermeras: Dilema Persistente para Lenguaje Común para Enfermeras: El Desafío de la Profesión.

El desafío pasa a ser la conquista de la profesión, ganando los conflictos y teniendo el coraje y la creatividad de buscar los medios para solucionar sus problemas, mostrando su contribución en la mejora de las condiciones de vida de la población.

Como vimos, el análisis del tema Un Lenguaje Común exige mucho más que la simple elaboración mecánica de un sistema de clasificación para llegar a tener un padrón de calidad.

La solidariedad, la cooperación dentro y fuera de profesión, y la unión de los miembros de la profesión son aspectos fundamentales de una acción transformadora.

Para llegar a esto es necesario tener otra perspectiva educacional en la educación de las enfermeras.

2 - Cambiar la Perspectiva Educacional de la Profesión de Enfermería: de Una Educación Represora para Una Educación Crítica, Libertadora.

La educación crítica consiste en ayudar a las personas o grupos a ser más conscientes de los factores que afectan su bienestar y descubrir su capacidad de cambiar y mejorar la situación y comportamiento individual y colectivo.

En la educación crítica, se colocan los problemas y los participantes desarrollan el poder de percibir críticamente la forma de su existencia en el mundo.

La visión del mundo no es más estático, pero una realidad en proceso de transformación.

El proceso de cuestionar y aprender es un proceso crítico, permitiendo a las 
personas ser pensadores críticos, pues estimula la creatividad de las personas para actuar dentro de una realidad.

La educación crítica es una actividad continua que se inicia con la relación del hombre con el medio ambiente. Uno de los elementos importantes de la educación crítica es el diálogo. Sin diálogo, no hay comunicación, y sin comunicación no hay una verdadera educación.

Con la educación critica posibilitar el desarrollar de la conciencia crítica, que es un proceso abierto de aprendizaje, que se da a través de debates.

La educación crítica exige el cambio de la orientación teórica de los currículos de enfermería.

3 - Cambiar el Paradigma que Orienta el Diseño de los Currículos La concepción filosófica de la educación crítica no puede ser operacionalizada en el diseño de currículos bajo el paradigma Salud-Enfermedad. Este paradigma es represor y enfatiza los aspectos negativos de la salud en detrimento de los positivos.

La educación crítica exige el paradigma de Promoción de la Salud, Nutrición y Desarrollo. La salud, la nutrición y el desarrollo son vistos dentro de la perspectiva socio ecológica. Así, se enfocan los aspectos positivos y potenciales de las relaciones del ser humano con la sociedad y el medio ambiente. Los aspectos negativos o los conflictos surgen del desequilibrio de esas relaciones. Lo importante es el desarrollo del potencial integral del ser humano, de la sociedad y del medio ambiente.

La actual Política de Salud de la OPS/OMS para América Latina está siendo desarrollada de acuerdo con esa orientación filosófica, aunque la correspondencia no siempre es explícita o exacta.

¿La enfermería tiene que comenzar a cuestionar sus referencias teóricos para saber qué dirección tomar para responder las preguntas básicas de quiénes somos, qué queremos, cómo lo queremos, y para quién?

4 - Cambiar la Perspectiva de la Práctica Cambiar la perspectiva de la práctica actual es adoptar una nueva visión de la salud, o sea, comprenderla como una condición socialmente adquirida. Las implicaciones de este cambio exigen redescubrir el papel de la enfermera y de su equipo de trabajo dentro de la estrategia de "Promoción de la Salud".

Actualmente las enfermeras están entrenadas para trabajar dentro de un sistema de salud que privilegia la "enfermedad", en detrimento de los aspectos positivos de la vida y de salud. Ellas no están debidamente preparadas para trabajar con "Promoción de la Salud". Para esto, los indicadores y criterios de los cuidados de enfermería tendrán que ser redefinidos y recreados dentro de los nuevos modelos de servicios de enfermería.

Llegó el momento de terminar la dicotomía entre lo académico y la práctica. El proceso de formación de la enfermera y su práctica deberá estar bajo un modelo único, para permitir la participación y cooperación de todo el equipo de enfermería. 
Quisiera terminar diciendo que los espacios están abiertos para los cambios. Necesitamos saber utilizarlos y mostrar nuestra real contribución en el sistema de salud de nuestros países y para la sociedad. Este camino va a exigir mucha solidariedad y persistencia, comenzando por las bases hacia las esferas más altas de la profesión.

Muchas gracias.

\section{A COMMON LANGUAGE TO NURSES: A PERSISTENT DILEMMA}

The author during her discussion tries to explain that the theme needs another approaching perspective. Therefore, she uses a critical model to find out the ideology of the theme; the factors that have contributed to the dilemma inside and outside the profession; presents a general view of the situation in Latin America and finalizes the paper with the indication of some actions aiming at transforming Nursing and enabling the discussion and work with this theme.

UNITERMS: common language; persistent dilemma; model; critical theory; ideology, transformation action.

\section{REFERENCIAS BIBLIOGRAFICAS}

01. AMERICAN NURSES ASSOCIATION. Nursing: a social policy statement. Kansas City: ANA, 1980. p. 2.

02. ASSOCIAÇÃO BRASILEIRA DE ENFERMAGEM. O ensino de pós-graduação em enfermagem no Brasil. Brasília: Cortez, 1989. p. 48. (Cadernos Científicos, 3).

03. BEAUVOIR, S. O segundo sexo: a experiência vivida. Rio de Janeiro: Nova Fronteira, 1980. p. 363.

04. BLOCH, D. The conceptualization of nursing research and nursing science. In: McCLOSHEY, J .C., GRACE, H.K. (eds). Current issues in nursing. 2. ed. Boston: Blackwell Scientific, 1985. p. 125-6.

05. CARPENITO, L. J. Diagnostico de enfermería: aplicación a la práctica clínica. Madrid: Interamericana/McGraw-Hill, 1987. p. 3. 
06. CONSEJO INTERNACIONAL DE ENFERMERÍAS (CIE). Manual sobre la reglamentación de enfermería: del principio al poder. Geneva: CIE, 1991. p. 43-7.

07. CONSEJO NACIONAL DE DESENVOLVIMENTO CIENTÍFICO E TECNOLÓGICO/CNPq. Avaliação e perspectivas. Brasília: CNPq, 1992. p. 176. v. 6 (Ciências da Saúde, Enfermagem, 38).

08. FREIRE, P. The politics of education: culture, power and liberation. Granby: Bregui \& Garvey, 1985. p. 75-6.

09. GOMEZ, P. Promoción de la salud: conceptos y estrategias. Washington: OPS/OMS, 1992. p. 5.

10. GORDON, M. Diagnostic category development. In: McCLOSHEY, J.C., GRACE, H.K. (eds). Current issues in nursing. Boston: Blackwell Scientific, 1985. p. 47.

11. HABERMAS, J. The public sphere: an encyclopedia article (1964). N. Ger. Crit., Boston, v. 1, n. 3, p. 49-55, 1974.

12. . Communication and the evolution of society. Boston: Beacon, 1979. p.50.

13. HODGES, L.C., ICENHOUR, M.L. Measuring the quality of nursing carc. In: MCCLOSHEY, J.C., GRACE, H.K. (eds). Current issues in nursing. Boston: Blackwell Scientific, 1985. p. 47.

14. MAINGÓN, T. Las políticas sociales: discusión teórica, conceptual y metodológica. Washington: OPS/OMS, 1992. p. 2 (Serie Informes Técnicos, 4).

15. MCCLOSKEY,J. C.,GRACE, H. K. Current issues in nursing. 3. ed. St. Louis: Mosby, 1990. p. 42: Defining and redefining nursing knowledge.

16. ORGANIZACIÓN MUNDIAL DE SALUD/ASOCIACIÓN CANADENSE DE SALUD PÚBLICA. Carta de Ottawa de promoción de salud. Ottawa: ACSP, 1986. p. 12.

17. ORGANIZACIÓN PANAMERICANA DE SALUD/ORGANIZACIÓN MUNDIAL DE SALUD. Lineamientos generales para el desarrollo de un programa de investigación en enfermería 1992-2000. In: COLOQUIO PANAMERICANO DE ENFERMERÍA, 3, 1992, Panamá. Anales. Panamá: OPS/OMS, 1992. p. 14.

18. Estudio sobre las tendencias de la práctica de enfermería en siete países de América Latina. Washington: OPS/OMS, 1989. (En revision) 19. - El movimiento de municipios saludables en América. Washington: OPS/OMS, 1992. p. 5-35.

20. POLIT, D., HUNGLER, B. Nursing research principles and methods. 2. ed. Filadelphia: Lippincott, 1983. p. 7-14.

21. WRIGHT, M.G.M. O espaço da mulher brasileira e o espaço da enfermeira brasileira. Rev.Gauch.Enfermagem, Porto Alegre, v. 5, n. 2, p. 345-56, 1984. 
22. . The need for a health-nutrition-development paradigm. /Trabajo presentado en el Instituto de Investigación en Salud y Enfermería, Copenhagen, 17 de junio, 1990./ 
FIGURA 1 - MODELO OPERACIONAL CRITICO PARA ANÁLISIS DEL TEMA: LENGUAJE COMÚN PARA ENFERMERAS (adaptando: Wright. M.G.M., Modelo Operacional Critico para la salud, 1990)

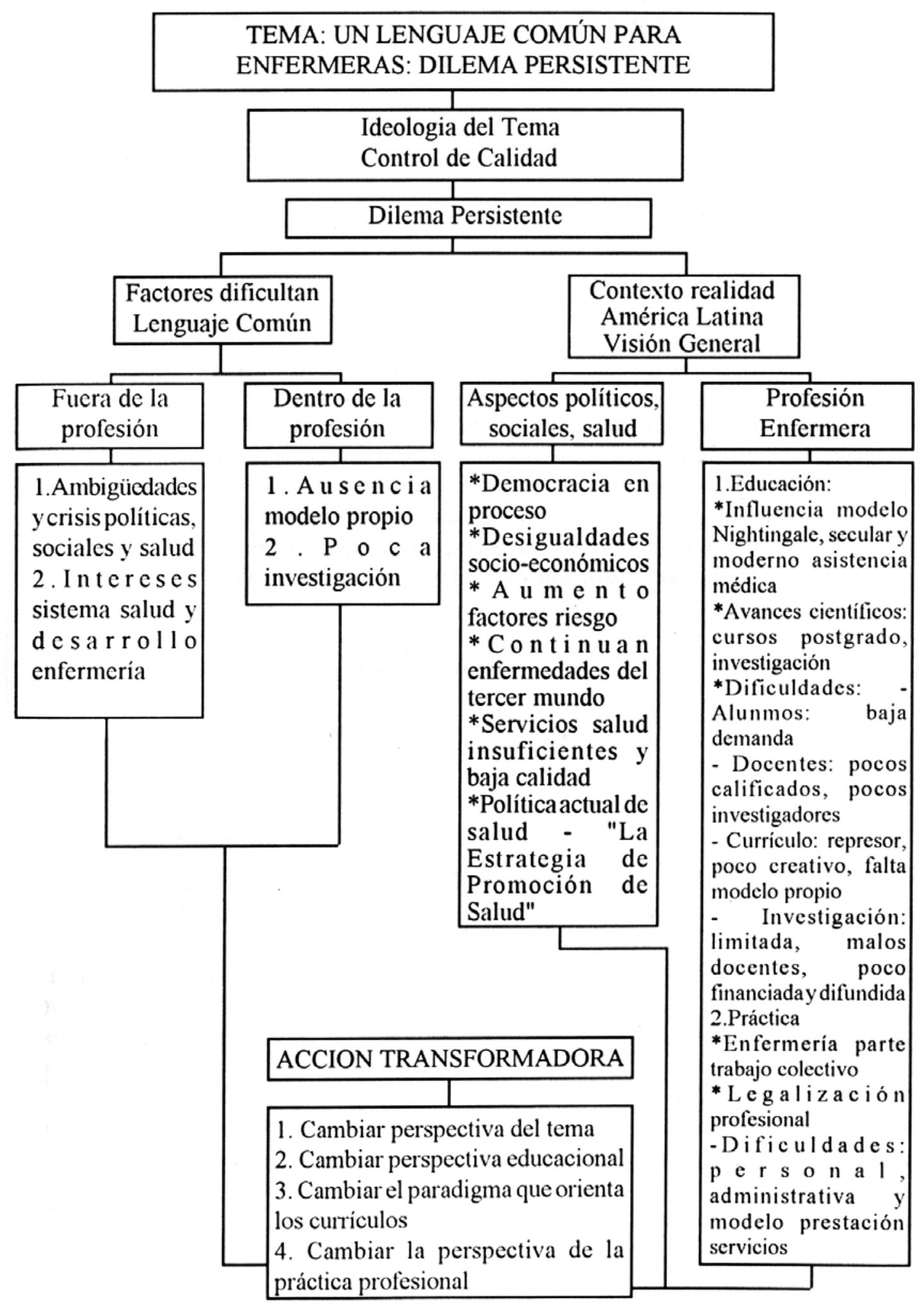

